\title{
Los vínculos que brindan mayor satisfacción en la red de apoyo social de los adultos mayores
}

\author{
Luciana Polizzi ${ }^{\star}$, Claudia Josefina Arias ${ }^{\star \star}$
}

* Licenciada en Psicología. Becaria de Perfeccionamiento, Universidad Nacional de Mar del Plata, Buenos Aires, Argentina.

Correo electrónico: polizzilu@gmail.com

** Magíster en Psicología Social. Profesora e investigadora de la Facultad de Psicología, Universidad Nacional de Mar del Plata, Buenos Aires, Argentina.

Correo electrónico:

cjarias@mdp.edu.ar

Recibido: 30 de abril del 2014

Aprobado: 3 de septiembre del 2014

Cómo citar este artículo: Polizzi, L. y Arias C. J. (2014). Los vínculos que brindan mayor satisfacción en la red de apoyo social de los adultos mayores. Pensando Psicología, 10(17), 61-70. doi: http://dx. doi. org/10.16925/pe.v10i17.785

\begin{abstract}
Resumen
Las redes de apoyo social constituyen un tema de gran interés en relación con los adultos mayores. Su efecto sobre el bienestar integral ha sido extensamente comprobado y por ello se ha incluido entre los principales objetivos, estrategias, planes y recomendaciones internacionales dirigidos a la población mayor. Este estudio se propuso tanto identificar y caracterizar los vínculos de la red de apoyo social que brindan mayor satisfacción en esta etapa de la vida, como evaluar las funciones de apoyo que estos cumplían según la conformación del grupo conviviente. Se implementó un diseño no experimental transversal. El estudio fue descriptivo-correlacional. Se seleccionó una muestra no probabilística-intencional de 120 adultos mayores (de 60 a 85 años), de ambos sexos y de tres tipos de grupo conviviente: los que vivían solos, los que vivían únicamente con su pareja y los que vivían con otros familiares. A la muestra se le administró el cuestionario de datos sociodemográficos y la Entrevista Estructurada de Fuentes de Apoyo Social (Vaux y Harrison, 1985). Los resultados evidencian el papel central que ocupan los hijos, la pareja y los amigos en la vejez. Además, se observaron diferencias estadísticamente significativas según género y grupo conviviente en la elección del vínculo que brinda mayor satisfacción.
\end{abstract}

Palabras clave: grupo conviviente, red de apoyo social, satisfacción, vejez.

Bonds that Provide the Most Satisfaction in Elderly People's Social Support Networks

\begin{abstract}
Social support networks are a subject of major interest in relation to elderly people. Their effect on allround wellbeing has been extensively shown and they have thus been included among the main objectives, strategies, plans and international recommendations directed towards the elderly population. This study aims to both identify and characterize the bonds of the social support network that provide the most satisfaction in this phase of life, and also to evaluate the support functions that these fulfil, according to the subject's living arrangements. A non-experimental, cross-sectional design was implemented for this descriptive-correlational study. A non-probability/intentional sample of 120 elderly people (aged from 60 to 85 ) was selected, of both sexes and from three types of living arrangements: living alone, living only with a partner, and living with other family members. A questionnaire of socio-demographic data and the Structured Interview of Social Support Sources (Vaux \& Harrison, 1985) was applied to the sample. The results show the central role that children, the partner and friends play in old age. Furthermore, there were statistically significant differences in the bond that gave the most satisfaction according to gender and living arrangements.
\end{abstract}

Keywords: living arrangements, social support network, satisfaction, old age.

Os vínculos que oferecem maior satisfação na rede de apoio social dos idosos

\section{Resumo}

As redes de apoio social constituem um tema de grande interesse quando se trata de idosos. Seu efeito sobre o bem-estar integral tem sido extensamente comprovado e, por isso, tem sido incluído entre os principais objetivos, estratégias, planos e recomendações internacionais dirigidos à população idosa. Este estudo se propõe identificar e caracterizar os vínculos da rede de apoio social que oferecem maior satisfação nessa etapa da vida, bem como avaliar as funções de apoio que estes cumprem segundo a conformação do grupo convivente. Implementou-se um desenho não experimental transversal. O estudo foi descritivo-correlacional. Selecionou-se uma amostra não probabilística-intencional de 120 idosos (de 60 a 85 anos), de ambos os sexos e de três tipos de grupo convivente: os que moravam sozinhos, os que moravam unicamente com seu/sua companheiro/a e os que moravam com outros familiares. Foram aplicados à amostra um questionário de dados sociodemográficos e a Entrevista Estruturada de Fontes de Apoio Social (Vaux e Harrison, 1985). Os resultados evidenciam o papel central que ocupam os filhos, o/a companheiro/a e os amigos da velhice. Além disso, observaram-se diferenças estatisticamente significativas segundo gênero e grupo convivente na escolha do vínculo que oferece maior satisfação.

Palavras-chave: grupo convivente, rede de apoio social, satisfação, velhice. 


\section{Introducción}

En la actualidad, las redes de apoyo social constituyen un tema de gran interés en relación con los adultos mayores. Los hallazgos de investigación han mostrado ampliamente su relación con el bienestar integral y la satisfacción vital en la vejez (Aguerre y Bouffard, 2008; Arias y Polizzi, 2013; Bishop, Martin y Poon, 2006; Fratiglioni, Paillard-Borg y Winblad, 2004; Kahn, Hessling y Russell, 2003; Phillips, Sui, Yeh y Cheng, 2008; Prince-Paul, 2008; Vanderhorst y McLaren, 2005; Vivaldi y Barra, 2012; Yoon y Lee, 2007; Zinbarg, Lee y Yoon, 2007). Las deficiencias en el apoyo social se han asociado con una amplia variedad de efectos adversos en la salud tanto física (Heckman, 2003; Uchino, 2006), como psicológica (Bishop, Kriegsman, Beekman y Deeg, 2004; Cacioppo, Hughes, Waite, Hawkley y Thisted, 2006; Dennis, Wakefield, Molloy, Andrews y Freidman, 2005; Garssen, 2004; O'Luanaigh y Lawlor, 2008; Reblin y Uchino, 2008; Waern, Rubenowitz y Wilhelmson, 2003) en las personas de edad avanzada.

El modelo teórico del apoyo social sostiene que la participación activa en los espacios sociales y la integración familiar y comunitaria incrementan el bienestar, elevan la calidad de vida y favorecen el empoderamiento (Iacub y Arias, 2011). Entre sus principales fundamentos podemos destacar que: 1) se centra en el estudio de los aspectos sociales, 2) posee un importante interés preventivo, 3) sostiene una concepción de salud positiva, 4) se interesa de manera fundamental por las problemáticas de personas en situación de fragilidad, 5) se centra en el trabajo con los recursos y las potencialidades, 6) persigue el logro de cambios a partir de la implicación activa de los involucrados en el problema y 7) apunta al potenciamiento y desarrollo tanto individual y grupal, como institucional y comunitario (Arias, 2005). Por estas razones, diversos organismos internacionales han otorgado prioridad al apoyo social incluyéndolo entre los principales objetivos de programas, estrategias y planes internacionales dirigidos a la población mayor (Naciones Unidas, 1998 y 2002; Organización Mundial de la Salud [OMs], 2002; Comisión Económica para América Latina y el Caribe [Cepal] y Centro Latinoamericano de Desarrollo Empresarial [Celade], 2002 y 2004).

La red de apoyo social pertenece a los apoyos informales y está conformada por un conjunto restringido de relaciones familiares y no familiares que brindan alguna o varias formas de ayuda. Se puede disponer de ella tanto de manera cotidiana como en situaciones críticas y resulta fundamentalmente útil para el desarrollo de soluciones creativas frente a conflictos y problemas.

La red puede ser evaluada en lo que respecta a las características estructurales, la funcionalidad y los atributos de los vínculos que la componen. Las características estructurales aluden a: tamaño de la red (cantidad de personas que la conforman), densidad (grado de interrelación entre los miembros), distribución (ubicación de las relaciones en los cuadrantes y círculos del mapa de red), dispersión (distancia espacial entre los miembros) y homogeneidad o heterogeneidad (similitud o diferencia de características sociales, culturales y demográficas entre los miembros de la red). En las funciones de la red se incluyen: compañía social, apoyo emocional, guía cognitiva y consejos y ayuda material, de servicios y acceso a nuevos contactos. Los atributos de los vínculos aluden a las funciones prevalecientes de estos, su multidimensionalidad (cantidad de funciones que cumple), reciprocidad, intimidad, frecuencia de los contactos e historia (Sluzki, 1996).

Las redes se modifican a lo largo de la vida y se ha propuesto que tienden a la disrupción durante la vejez, llegando en algunos casos a situaciones extremas que provocan el aislamiento social del adulto mayor. Según Arias (2009), este supuesto ha sido extensamente aceptado, considerándose como reglas generales que durante la vejez: a) la red sufría importantes pérdidas, b) que se dificultaba tanto el mantenimiento de antiguos vínculos como la incorporación de nuevas relaciones y c) que, como consecuencia de lo anterior, los adultos mayores disponían de escasos recursos de apoyo social, sufriendo muy frecuentemente aislamiento y soledad, así como patologías derivadas de ello (Sluzki, 1996).

Sin embargo, en la actualidad, el supuesto de disrupción está siendo cuestionado a partir de los hallazgos de investigaciones que han demostrado que los adultos mayores poseen redes amplias que incorporan nuevos vínculos durante esta etapa de la vida y que se encuentran satisfechos con el apoyo del que disponen (Arias, 2004; Arias 2013a; Arias 2013b; Arias y Iacub, 2013; Cornwell, Schumm, Laumann y Graber, 2009; Fernández-Ballesteros, Reig Ferrer y Zamarrón, 2009). Por otro lado, se ha destacado que, si bien algunos hechos como la muerte de familiares y de amigos, la migración en la vejez, la jubilación y el ingreso a una residencia pueden impactar negativamente sobre las redes, estas presentan una amplia variabilidad durante la vejez evidenciando que la edad por sí sola no implica aislamiento y soledad, ni determina la insuficiencia de los apoyos sociales de un modo inexorable (Arias, 2009). 
En esta dirección, existen investigaciones que al comparar los tamaños de las redes por grupo de edad no han encontrado diferencias significativas entre estos (Arias, Botas y Polizzi, 2011; Cornwell et al., 2009). Otro estudio realizado en diferentes grupos etarios muestra que, mientras las relaciones positivas se mantienen estables, las negativas se reducen en la medida en que aumenta la edad (Akiyama, Antonucci, Takahashi y Langfahl, 2003). La teoría de la selectividad socioemocional (Carstensen, Isaaowitz y Charles, 1999; Carstensen, Pasupathi, Mayr y Nesselroade, 2000; Carstensen y Charles, 2007) sostiene que los adultos mayores asumen un rol proactivo en sus relaciones sociales y seleccionan intencionada y estratégicamente los vínculos que más les interesan seguir manteniendo y profundizando.

Los hijos, la pareja y los amigos son los vínculos que han sido identificados como los que brindan mayor satisfacción (Arias, 2004; Domínguez, Espín y Bayorre, 2001; Salinas, Manrique y Téllez-Rojo, 2008). Algunos estudios han mostrado que aquellos que viven con sus familias presentan niveles de salud mental, física y emocional más elevados que aquellos que viven solos (Bozo, Toksabay y Kürüm, 2009; Vivaldi y Barra, 2012). Otros estudios dan cuenta de que para la sintomatología depresiva, la ausencia de familiares cuando se dispone del apoyo de amigos es menos perjudicial que la ausencia de amigos cuando se dispone del apoyo de familiares (Fiori, Antonucci y Cortina, 2006).

En el ámbito familiar, las investigaciones muestran que los hombres informan recibir más apoyo de sus parejas (Arias y Polizzi, 2013; Reyes, Camacho, Eschbach y Markides, 2006), mientras que las mujeres tienden a priorizar a los hijos como suministradores de apoyo (Meléndez-Moran, Tomás-Miguel y Navarro-Pardo, 2007).

Respecto a la relación de amistad, las investigaciones ponen énfasis en la importante función socializadora que tiene este vínculo (Arias, 2013a; Arias, 2013b). Los estudios dan cuenta de que las buenas amistades producen un aumento en la longevidad de las personas (Giles, Glonek, Luszcz y Andrews, 2005) y reducen el riesgo de morir por cualquier causa (Shirom, Toker, Alkaly, Jacobson y Balicer, 2011).

En esta dirección, y profundizando la evaluación de la red en los adultos mayores, los objetivos de este estudio apuntan tanto a identificar y caracterizar dentro de la red los vínculos que brindan mayor satisfacción a los adultos mayores según grupo conviviente, como a conocer a qué tipos de vínculos (hijos, pareja, amigos, hermanos, etcétera) corresponden con mayor frecuen- cia y si son predominantemente familiares y del mismo género que los adultos mayores entrevistados.

\section{Metodología}

Se implementó un diseño de tipo no experimental transversal-correlacional. Se seleccionó una muestra no probabilística de tipo intencional de 120 adultos mayores de la ciudad de Mar del Plata (que no tuvieran deterioro cognitivo aparente) de tres tipos de grupo conviviente: 1) los que vivían solos, 2) los que vivían únicamente con su pareja y 3) los que vivían con otros familiares que no fuera la pareja. La tabla 1 muestra cómo quedó constituida la muestra.

Tabla 1

Conformación de la muestra según género y grupo conviviente

\begin{tabular}{lll}
\hline Grupo conviviente & $\begin{array}{c}\text { Adultos mayores } \\
\text { varones }\end{array}$ & $\begin{array}{c}\text { Adultas mayores } \\
\text { mujeres }\end{array}$ \\
\hline Vive solo & 20 & 20 \\
$\begin{array}{l}\text { Unicamente con la } \\
\text { pareja }\end{array}$ & 20 & 20 \\
$\begin{array}{l}\text { Con otro familiar } \\
\text { (no con pareja) }\end{array}$ & 20 & 20 \\
Total & 60 & 60 \\
\hline
\end{tabular}

Nota. Elaboración propia.

En un único encuentro, se administraron de manera oral e individual los siguientes instrumentos de recolección de datos:

Cuestionario de datos sociodemográficos: explora el sexo, la edad, el nivel de instrucción, si estaba jubilado, el tipo de hogar y las actividades principales que desarrollaba.

Entrevista estructurada de fuentes de apoyo social (Social support resources de Vaux y Harrison, 1985): esta ha sido aplicada con población adulta mayor residente en la Argentina, mostrando adecuadas propiedades psicométricas (Arias, 2004; Arias y Polizzi, 2013). Evalúa cinco formas de apoyo: emocional, práctico, financiero, social y de orientación y consejo. Además, proporciona información sobre las características estructurales y los atributos de los vínculos. Se pide al entrevistado que nombre a las personas que le brindan los distintos tipos de apoyo describiéndoles situaciones típicas a modo de ejemplificaciones que faciliten la identificación de las relaciones en cuestión. Una vez detectadas las personas que cumplen las diferentes funciones de apoyo, se elabora el listado total de relaciones que posee la persona y se 
completa una serie de datos de cada una de ellas que permiten identificar de manera específica el tipo de vínculo que se posee con cada uno de los miembros de la red.

Asimismo, al finalizar la entrevista estructurada, se incluyó una pregunta que exploraba qué vínculo de la red le brindaba mayor satisfacción a las personas entrevistadas.

Durante el proceso de recolección de datos, las personas que formaron parte de la muestra firmaron el "Consentimiento Informado" luego de haber recibido las explicaciones pertinentes. En este se explicitan los objetivos de la investigación y se aclara que la participación es voluntaria, anónima, confidencial y que la información revelada se utilizará con fines exclusivamente científicos, bajo la jurisdicción de la Ley Nacional 25326 de protección de los datos personales.

Los datos fueron analizados de manera cuantitativa. Con el fin de explorar las relaciones existentes entre la red de apoyo social, el género y el grupo conviviente, se aplicaron técnicas de estadística descriptiva e inferencial mediante la utilización del paquete estadístico informatizado InfoStat.

\section{Resultados}

El promedio de edad de las personas que conformaron la muestra fue de 73,75 años $(\mathrm{DE}=5,26)$. En relación con el estado civil, 49 son casados o tienen pareja, 49 son viudos, 11 separados y 11 solteros. El nivel educativo estuvo distribuido del siguiente modo: 41 tienen estudios primarios (33 completos y 8 incompletos), 49 estudios secundarios (33 completos y 16 incompletos), 11 estudios terciarios y 19 estudios universitarios (10 completos y 9 incompletos). En relación con las personas que integran el hogar, 40 viven en pareja, 40 en familia (esto incluía la convivencia con hijos en 29 casos, hermanos en 5 , con padre o madre en 5 casos y con sobrino en un caso) y 40 solos. En lo referido a la jubilación, 111 refirieron estar jubilados y 9 no. ${ }^{1}$

\footnotetext{
1 Cabe destacar que el amplio porcentaje de personas mayores que contaban con jubilación dentro de la muestra refleja la situación de la población en estudio. En Argentina existen diversas políticas nacionales que protegen los derechos de los adultos mayores. Entre ellas se encuentran la incorporación de jubilaciones no contributivas y para amas de casas que ampliaron la cobertura tanto en lo referido a seguridad social como médica al 95\% de los adultos mayores del país. Asimismo, existen variados programas nacionales que brindan una oferta amplia de actividades que propician la integración de las personas mayores y promueven su bienestar. Estas políticas públicas han contribuido al logro de baja incidencia de pobreza y mejores condiciones de vida en dicho grupo de la población (Roqué y Fassio, 2012; Arias, 2013a).
}

\section{Identificación de los vínculos que brindan mayor satisfacción}

Entre los vínculos que brindan mayor satisfacción, se destacaron predominantemente los hijos, siguiéndole en orden decreciente los amigos, otros familiares como nietos, hermanos, sobrinos, cuñadas y nueras, y por último las parejas (tabla 2).

Teniendo en cuenta el grupo conviviente de las personas entrevistadas, la prueba de $\mathrm{x}^{2}$ mostró existencia de relación significativa con el vínculo que brinda mayor satisfacción. Se puede observar que los hijos fueron mencionados con mayor frecuencia por los adultos mayores que viven con algún familiar; las parejas por aquellos que viven con ella, y finalmente los amigos y otros familiares por aquellos que viven solos (tabla 3).

La prueba $\mathrm{x}^{2}$ mostró existencia de relación significativa entre el género de las personas entrevistadas y los vínculos que brindan mayor satisfacción. Si bien los hijos son los más mencionados tanto por las mujeres como por los varones, mientras las mujeres eligen en segundo lugar a otros familiares, luego a los amigos y por último a su pareja, los hombres optan por los amigos y la pareja en igual medida, y por último por otros familiares (tabla 4).

\section{Caracterización de los vínculos que brindan mayor satisfacción}

Los vínculos que brindan mayor satisfacción fueron en su mayoría familiares. Esto pudo observarse tanto al analizar la muestra total como al hacerlo por grupo conviviente. En ambos casos, los vínculos familiares elegidos fueron principalmente los hijos y la pareja (tablas 5 y 6 ).

Como se puede observar en la tabla 7, los vínculos que brindan mayor satisfacción son predominantemente del mismo género que los adultos mayores entrevistados. Se observó que de las 60 mujeres entrevistadas, 46 mencionaron a personas de su mismo sexo; y contrariamente, de los 60 hombres, sólo 27 eligió a individuos de su mismo sexo. De esta manera, las mujeres fueron consideradas en mayor medida que los hombres como vínculo que brinda mayor satisfacción independientemente del género de quien elige. La prueba de $\mathrm{x}^{2}$ mostró la existencia de una relación significativa entre el género de las personas entrevistadas y el de los vínculos que brindan mayor satisfacción. 
Tabla 2

Vinculos que brindan mayor satisfacción para las personas mayores entrevistadas

\begin{tabular}{|c|c|c|c|c|c|c|c|c|c|}
\hline \multicolumn{2}{|c|}{ Hijos } & \multicolumn{2}{|c|}{ Amigos } & \multicolumn{2}{|c|}{ Otros familiares $^{\mathrm{a}}$} & \multicolumn{2}{|c|}{ Pareja } & \multicolumn{2}{|c|}{ Total } \\
\hline N. ${ }^{\circ}$ & $\%$ & $\mathrm{~N} .^{\circ}$ & $\%$ & $\mathrm{~N} .^{\circ}$ & $\%$ & N. ${ }^{\circ}$ & $\%$ & $\mathrm{~N} .^{\circ}$ & $\%$ \\
\hline 50 & 41,7 & 28 & 23,3 & 22 & 18,3 & 20 & 16,7 & 120 & 100,0 \\
\hline
\end{tabular}

Nota. Elaboración propia.

a 8 nietos, 4 hermanos, 3 sobrinos, 2 cuñados, 2 nueras, 1 madre, 1 bisnieto y 1 primo.

Tabla 3

Vínculos que brindan mayor satisfacción para las personas mayores entrevistadas según grupo conviviente

\begin{tabular}{lccccccccccc}
\hline \multirow{2}{*}{ Grupo conviviente } & \multicolumn{2}{c}{ Hijos } & \multicolumn{2}{c}{ Pareja } & \multicolumn{2}{c}{ Amigos } & Otros familiares & \multicolumn{2}{c}{ Total } \\
\cline { 2 - 12 } & N. & $\%$ & N. ${ }^{\circ}$ & $\%$ & N. ${ }^{\circ}$ & $\%$ & N. $^{\circ}$ & $\%$ & N. $^{\circ}$ & $\%$ \\
\hline Familia & 21 & 52,5 & 1 & 2,5 & 7 & 17,5 & 11 & 27,5 & 40 & 100 \\
Solo & 17 & 42,5 & 6 & 15,0 & 12 & 30,0 & 5 & 12,5 & 40 & 100 \\
Con pareja & 12 & 30,0 & 13 & 32,5 & 9 & 22,5 & 6 & 15,0 & 40 & 100 \\
\hline
\end{tabular}

Nota. $x^{2}=0,008 p<0,01$. Elaboración propia.

Tabla 4

Vínculos que brindan mayor satisfacción para las personas mayores entrevistadas según género

\begin{tabular}{|c|c|c|c|c|c|c|c|c|c|c|}
\hline \multirow[t]{2}{*}{ Género } & \multicolumn{2}{|c|}{ Hijos } & \multicolumn{2}{|c|}{ Pareja } & \multicolumn{2}{|c|}{ Amigos } & \multicolumn{2}{|c|}{ Otros familiares } & \multicolumn{2}{|c|}{ Total } \\
\hline & $\mathrm{N} .^{\circ}$ & $\%$ & $\mathrm{~N} .^{\circ}$ & $\%$ & N. ${ }^{\circ}$ & $\%$ & $\mathrm{~N} .^{\circ}$ & $\%$ & N. ${ }^{\circ}$ & $\%$ \\
\hline Femenino & 23 & 38,4 & 6 & 10,0 & 14 & 23,3 & 17 & 28,3 & 60 & 100 \\
\hline Masculino & 27 & 45,1 & 14 & 23,3 & 14 & 23,3 & 5 & 8,3 & 60 & 100 \\
\hline Total & 50 & 41,7 & 20 & 16,7 & 28 & 23,3 & 22 & 18,3 & 120 & 100 \\
\hline
\end{tabular}

Nota. $x^{2}=0,018 p<0,05$. Elaboración propia.

Tabla 5

Tipo de vínculo familiar-no familiar que brinda mayor satisfacción a las personas mayores entrevistadas

\begin{tabular}{lcc}
\hline $\begin{array}{c}\text { Tipo de vínculo que brinda } \\
\text { mayor satisfacción }\end{array}$ & N. & $\%$ \\
\hline Familiares & 92 & 76,7 \\
No familiares & 28 & 23,3 \\
Total & 120 & 100 \\
\hline
\end{tabular}

Nota. Elaboración propia.

Tabla 7

Género de los vínculos que brindan mayor satisfacción en las personas entrevistadas en hombres y mujeres

\section{Género del vínculo que brinda mayor satisfacción}

\begin{tabular}{|c|c|c|c|c|c|c|}
\hline \multirow{2}{*}{$\begin{array}{l}\text { Género de los adultos } \\
\text { mayores entrevistados }\end{array}$} & \multicolumn{2}{|c|}{ Femenino } & \multicolumn{2}{|c|}{ Masculino } & \multicolumn{2}{|c|}{ Total } \\
\hline & N. ${ }^{\circ}$ & $\%$ & $\mathrm{~N} .^{\circ}$ & $\%$ & N. ${ }^{\circ}$ & $\%$ \\
\hline Femenino & 46 & 76,7 & 14 & 23,3 & 60 & 100 \\
\hline Masculino & 33 & 55,0 & 27 & 45,0 & 60 & 100 \\
\hline Total & 79 & 65,8 & 41 & 34,2 & 120 & 100 \\
\hline
\end{tabular}

Nota. $x^{2}=0,012 p<0,05$. Elaboración propia. 


\section{Atributos de los vínculos que brindan mayor satisfacción}

Respecto al nivel de intimidad de los vínculos que brindan mayor satisfacción, se pudo observar que casi la totalidad $(96,6 \%)$ fueron mencionados como vínculos extremadamente íntimos y muy íntimos. En ningún caso fueron considerados como poco cercanos (tabla 8).

Tabla 8

Nivel de intimidad de los vínculos que brindan mayor satisfacción para las personas mayores entrevistadas

\begin{tabular}{lcc}
\hline \multicolumn{1}{c}{ Nivel de intimidad } & N. ${ }^{\circ}$ & $\%$ \\
\hline Extremadamente cercano & 100 & 83,4 \\
Muy cercano & 16 & 13,3 \\
Bastante cercano & 4 & 3,3 \\
Poco cercano & 0 & 0,0 \\
Total & 120 & 100 \\
\hline
\end{tabular}

Nota. Elaboración propia.

Asimismo, respecto de la frecuencia de contacto, en la tabla 9 se puede observar que las personas mayores se contactan con gran periodicidad con las personas que les brindan mayor satisfacción mediante visitas o comunicación telefónica. En el 90,8\% de los casos, el contacto se establece al menos con una frecuencia semanal.

Tabla 9

Frecuencia de contacto con los vínculos que brindan mayor satisfacción para las personas mayores entrevistadas

\begin{tabular}{lcc}
\hline \multicolumn{1}{c}{$\begin{array}{c}\text { Frecuencia de } \\
\text { contacto }\end{array}$} & N. ${ }^{\circ}$ & $\%$ \\
\hline Casi todos los días & 71 & 59,3 \\
Dos veces por & 19 & 15,8 \\
semana & 19 & 15,8 \\
Una vez por semana & 4 & 3,3 \\
Cada 15 días & 7 & 5,8 \\
Una vez al mes o & & \\
menos & 120 & 100 \\
Total & & \\
\hline
\end{tabular}

Nota. Elaboración propia.

Teniendo en cuenta la historicidad de los vínculos que brindan mayor satisfacción, la mayoría de estos habían sido conocidos hacía más de 20 años por las personas entrevistadas (tabla 10).
Tabla 10

Historicidad del vínculo que brinda mayor satisfacción para las personas mayores entrevistadas

\begin{tabular}{lcc}
\hline $\begin{array}{c}\text { Historia } \\
\text { del vínculo }\end{array}$ & $\mathrm{N}^{\circ}{ }^{\circ}$ & $\%$ \\
\hline 20 años o más & 104 & 86,7 \\
Entre 15 y 8 años & 10 & 8,3 \\
Menos de 8 años & 6 & 5,0 \\
Total & 120 & 100 \\
\hline
\end{tabular}

Nota. Elaboración propia.

Respecto a la cantidad de funciones de apoyo que brindan estos vínculos a las personas entrevistadas, se pudo observar que el 78,3\% cumplía dos o más funciones, constituyendo vínculos multidimensionales. El resto cumplía sólo una de las cinco funciones de apoyo posibles (tabla 11).

Tabla 11

Multidimensionalidad de los vínculos que brindan mayor satisfacción para las personas mayores entrevistadas

\begin{tabular}{lcc}
\hline $\begin{array}{c}\text { Multidimensionalidad de los vínculos que } \\
\text { brindan mayor satisfacción }\end{array}$ & N. ${ }^{\circ}$ & $\%$ \\
\hline Dos o más funciones & 94 & 78,3 \\
Sólo una función & 26 & 21,7 \\
Total & 120 & 100 \\
\hline
\end{tabular}

Nota. Elaboración propia.

Centrándonos en las funciones de apoyo que cumplen predominantemente los vínculos que brindan mayor satisfacción a los adultos mayores, se observó que la mayoría de las personas manifiestan que los apoyos preponderantes son el emocional y la compañía social. Le siguieron la ayuda financiera, el consejo o guía cognitiva y la ayuda práctica en orden de frecuencia decreciente (tabla 12).

Tabla 12

Funciones de apoyo que cumplen los vínculos que brindan mayor satisfacción para las personas mayores entrevistadas

\begin{tabular}{lcccccc}
\hline $\begin{array}{c}\text { Funciones de } \\
\text { los vínculos que } \\
\text { brindan mayor } \\
\text { satisfacción }\end{array}$ & \multicolumn{2}{c}{ Sí } & \multicolumn{2}{c}{ No } & \multicolumn{2}{c}{ Total } \\
\cline { 2 - 7 } & N. ${ }^{\circ}$ & $\%$ & N. $^{\circ}$ & $\%$ & N. ${ }^{\circ}$ & $\%$ \\
\hline Apoyo emocional & 91 & 75,8 & 29 & 24,2 & 120 & 100 \\
Compañía social & 90 & 75,0 & 30 & 25,0 & 120 & 100 \\
Ayuda financiera & 48 & 40,0 & 72 & 60,0 & 120 & 100 \\
Consejo-guía & 47 & 39,2 & 73 & 60,8 & 120 & 100 \\
Ayuda práctica & 36 & 30,0 & 84 & 70,0 & 120 & 100 \\
\hline
\end{tabular}

Nota. Elaboración propia. 


\section{Discusión}

Los resultados alcanzados muestran el papel central que ocupan durante la vejez los hijos, la pareja y los amigos. Estos hallazgos concuerdan con los obtenidos en investigaciones anteriores y refuerzan la importancia que poseen en esta etapa de la vida (Arias, 2004; 2005; Arias et al., 2011). Dichos vínculos tienen la característica de ser mayoritariamente multidimensionales, es decir, que cumplen dos o más funciones de apoyo, entre las que se encuentran fundamentalmente la emocional y la social. La valoración de estas relaciones como extremadamente íntimas o muy íntimas, en casi la totalidad de los casos, muestra que son vínculos que poseen características de mucha cercanía y confidencialidad. Por su parte, la elevada frecuencia de contacto con ellos posibilita que los apoyos que brindan estas personas estén disponibles y puedan ser efectivos cotidianamente.

Teniendo en consideración el grupo conviviente de las personas entrevistadas, se pueden observar diferencias significativas en lo que hace a la elección del vínculo que brinda mayor satisfacción. Si bien los hijos fueron, en términos generales, los más elegidos por todos, aquellos adultos mayores que vivían con algún familiar los mencionaron con mayor frecuencia. Asimismo, los amigos fueron globalmente los segundos más elegidos, fundamentalmente por aquellos que vivían solos. El tercer vínculo más nombrado fue la pareja, y estas fueron elegidas mayoritariamente por aquellos que vivían con ellas. Se puede apreciar que, de acuerdo con el grupo conviviente, los adultos mayores optan por diferentes relaciones como las que brindan mayor satisfacción. Esto puede deberse fundamentalmente al tipo de relaciones que se encuentran o no disponibles de acuerdo con su situación actual.

De los resultados se desprenden, además, diferencias de género que implican principalmente que los hombres elijan más a sus parejas en comparación con las mujeres, y que estas incluyan más a otros familiares como nietos, hermanas, cuñadas o nueras, entre otros. Esto se puede pensar a la luz de otra diferencia significativa que se observa en los resultados, a saber: que los vínculos femeninos fueron los más mencionados, tanto por mujeres como por hombres, como los que brindan mayor satisfacción. Estos hallazgos pueden ser interpretados a partir de la existencia de condicionantes familiares y culturales relacionados con mandatos $y$ roles asignados de manera diferencial a los varones y a las mujeres. Existe una amplia evidencia que muestra que las mujeres son, de un modo generalizado, las que a lo largo de su vida se ocupan más frecuentemente de las tareas de cuidado (Huenchuan, Roqué y Arias, 2009; Marco-Navarro, 2006; Robles, 2003). Una investigación reciente realizada con personas heterosexuales muestra que los hombres valoraban a sus parejas como proveedoras de apoyo emocional y de compañía social (Arias y Polizzi, 2013). Si consideramos que estas dos funciones son las características principales que se atribuyen a los vínculos que brindan mayor satisfacción, resulta claro que las mujeres sean mayoritariamente elegidas que los hombres. Como se expresó anteriormente, esto puede entenderse como consecuencia de procesos de socialización diferenciales en los que se consideran estas habilidades como inherentes a la función femenina y se refuerza su desarrollo en ellas (Arias y Polizzi, 2013).

En estudios anteriores se pudo observar que los adultos mayores incorporan nuevos vínculos - tanto familiares como no familiares- durante esta etapa de la vida, y si bien se producen pérdidas, la inclusión de nuevas relaciones logra compensarlas y mantener el tamaño de la red (Arias y Polizzi, 2010; Arias, 2013b). Esto no sólo desestima el supuesto de aislamiento social, sino que a su vez apoya las afirmaciones de la teoría de la selectividad socioemocional (Carstensen et al., 1999; Carstensen et al., 2000; Carstensen y Charles, 2007) que definen al adulto mayor como un ser proactivo en la búsqueda y el mantenimiento de sus relaciones sociales. Sin embargo, resulta importante resaltar que los vínculos que brindan mayor satisfacción fueron en su gran mayoría aquellas relaciones de más de 20 años, por lo que tal vez la compensación entre incorporación y pérdida se produzca en lo referido a la cantidad de vínculos que conforman la red pero no en lo que respecta a sus atributos. Se podría pensar que los vínculos incorporados más recientemente no posean la misma funcionalidad o multidimesionalidad que los que tienen más historia. Por tal motivo, en futuras investigaciones sería interesante centrar la exploración en los vínculos nuevos con el fin de conocer sus atributos de nivel de intimidad, frecuencia de contacto, multidimensionalidad y funciones de apoyo que cumplen predominantemente. Esto efectuará aportes que permitirán evaluar si después de las pérdidas e incorporaciones de vínculos que se producen en la vejez, la red sigue satisfaciendo al sujeto en la misma medida en que lo hacía antes que estos cambios se produjeran. 


\section{Conclusiones}

Los resultados de esta investigación son consistentes con hallazgos de estudios efectuados desde la psicología positiva que aportan evidencia acerca de que en la vejez no sólo hay déficits, pérdidas y deterioro.

Como se pudo observar, las personas mayores poseen vínculos importantes que les brindan no sólo apoyo, sino también elevados niveles de satisfacción. Sin embargo, hay que tener en cuenta los alcances de estos resultados, ya que corresponden a una muestra reducida de una población que tiene sus características particulares. Como se ha señalado anteriormente, la población de personas mayores en la República Argentina posee casi en su totalidad cobertura previsional y médica y gran parte de ellos participan en actividades en la comunidad. Futuras investigaciones que se enfoquen en poblaciones de adultos mayores más vulnerables y con mayores desventajas sociales o económicas permitirán constatar si los resultados son similares o no respecto de los logrados en este estudio. Por otra parte, como se expuso anteriormente, resulta interesante continuar explorando los cambios que se producen en la vejez por pérdidas e incorporación de vínculos, con el fin de identificar si esta logra satisfacer al sujeto en la misma medida en que lo hacía antes que estos se produjeran. Lograr avances en dicho sentido sería importante para valorar si las personas mayores continúan disponiendo de los apoyos suficientes que respondan de manera satisfactoria a sus necesidades tanto emocionales como instrumentales.

\section{Referencias}

Aguerre, C. y Bouffard, L. (2008). Envejecimiento exitoso: teorías, investigaciones y aplicaciones clínicas. Revista de la Asociación Colombiana de Gerontología y Geriatría, 22, 1146-1162.

Akiyama, H., Antonucci, T. K., Takahashi, K. y Langfahl, E. S. (2003). Negative Interactions in Close Relationships across the Life Span. Journals of Gerontology Series BPsychological Sciences and Social Sciences, 58(2), 70-79. doi: 10.1093/geronb/58.2.P70

Arias, C. (2004). Red de apoyo social y bienestar psicológico en personas de edad. Mar del Plata: Suárez.

Arias, C. (2005). Composición de la Red de Apoyo Social de Adultos Mayores: vínculos familiares y no familiares. En L. Golpe y C. Arias (Eds.), Sistemas formales e informales de apoyo social para los adultos mayores: aportes de una investigación científica al campo de la gerontología institucional (pp. 233-249). Mar del Plata: Suárez.

Arias, C. (2009). La red de apoyo social. Evaluación e intervención con personas adultas mayores. Revista de Psicología de IMED, 1(1), 147-158. Recuperado de http:// www.imed.br/revistapsico

Arias, C. (2013a). Aportes del apoyo social en el delineamiento de políticas públicas para las personas mayores. En V. Montes de Oca (Coord.), Envejecimiento en América Latina y el Caribe: enfoques en investigación en envejecimiento (pp. 425-452). México: Instituto de Investigaciones Sociales, UNAM.

Arias, C. (2013b). El apoyo social en la vejez: la familia, los amigos y la comunidad. Revista Kairós Gerontologia, 16(6), 25-40.

Arias, C. Botas, N. y Polizzi, L. (2011). Características de la red de apoyo social: comparación entre adultos jóvenes y adultos mayores residentes en la ciudad de Mar del Plata. Ponencia presentada en el xI Congreso Virtual de Psiquiatría Interpsiquis. Recuperado de http://www.psiquiatria.com/congreso/2011/psicologia/articulos/50166/

Arias, C. y Iacub, R. (2013). ¿Por qué investigar aspectos positivos en la vejez? Contribuciones para un cambio de paradigma. Publicatio UEPG: Ciências Humanas, Linguística, Letras e Artes, 21(2), 271-281. doi: 10.5212/ PublicatioHuma.v.21i2.00012

Arias, J. C. y Polizzi, L. (2010). La red de apoyo social en la vejez: pérdidas e incorporación de nuevos vínculos. Ponencia presentada en el x Congreso Virtual de Psiquiatría Interpsiquis. Recuperado de http://www.psiquiatria.com/bibliopsiquis/handle/10401/934

Arias, J. C. y Polizzi, L. (2013). The Couple Relationship Support Functions and Sexuality in Old Age. Journal Kairós Gerontologia, 16(1), 27-48.

Bishop, A. J., Martin, P. y Poon, L. (2006). Happiness and Congruence in Older Adulthood: A Structural Model of Life Satisfaction. Aging and Mental Health, 10(5), 445-453.

Bishop, M. I., Kriegsman, D. M. W., Beekman, A. T. F. y Deeg, D. J. H. (2004). Chronic Diseases and Depression: The Modifying Role of Psychosocial Resources. Social Science and Medicine, 59, 721-733.

Bozo, O., Toksabay, N. y Kürüm, O. (2009). Activities of Daily Living, Depression, and Social Support among Elderly Turkish People. Journal of Psychology, 143, 193-205.

Cacioppo, J. T., Hughes, M. E., Waite, L. J., Hawkley, L. C. y Thisted, R. A. (2006). Loneliness as a Specific Risk Factor for Depressive Symptoms: Cross-Sectional and Longitudinal Analyses. Psychol Aging, 21, 140-151.

Carstensen, L., Isaaowitz, D. y Charles, S. T. (1999). Taking Time Seriously in Life Span Development. American Psychologist, 54, 165-181. 
Carstensen, L., Pasupathi, M., Mayr, U. y Nesselroade, J. (2000). Emotional Experience in Everyday Life across the Adult Life Span. Journal of Personality and Social Psychology, 79(4), 644-655. doi: 10.1037/00223514.79.4.644

Carstensen, L. y Charles, S. T. (2007). El envejecimiento humano: ¿por qué incluso las buenas noticias se toman como malas? En L. G. Aspinwall y U. M. Staudinger (Eds.), Psicología del potencial humano: cuestiones fundamentales y normas para una psicología positiva (pp. 111-126). Barcelona: Gedisa.

Comisión Económica para América Latina y el Caribe (CEPAL) y Centro Latinoamericano de Desarrollo Empresarial (CELADE). (2002). Recomendaciones para políticas sobre redes de apoyo social de personas adultas mayores. Santiago de Chile: CEPAL-CELADE.

Comisión Económica para América Latina y el Caribe (CEPAL) y Centro Latinoamericano de Desarrollo Empresarial (CELADE). (2004). Estrategia regional e implementación para América Latina y el Caribe del Plan de Acción Internacional de Madrid sobre el Envejecimiento. Santiago de Chile: CEPAL-CELADE.

Cornwell, B., Schumm, L. P., Laumann, E. O. y Graber, J. (2009). Social Networks in the nshap Study: Rationale Measurement and preliminary Findings. Journal of Gerontology: Social Sciences, 64(1), 47-55. doi: 10.1093/ gerontob/gbp042

Dennis, M., Wakefield, P., Molloy, C., Andrews, H. y Freidman, T. (2005). Self-Harm in Older People with Depression: Comparison of Social Factors, Life Events and Symptoms. Br J Psychiatry, 186, 538-539.

Domínguez, T., Espín, A. y Bayorre, H. (2001). Caracterización de las relaciones familiares del anciano. Revista Cubana de Medicina General Integral, 17, 418-422.

Fernández-Ballesteros, R., Reig Ferrer, A. y Zamarrón, M. D. (2009). Evaluación en psicogerontología. En R., Fernández Ballesteros (Ed.), Psicología de la vejez: una psicogerontología aplicada (pp. 35-78). Madrid: Pirámides.

Fiori, K. L., Antonucci, T. C. y Cortina, K. S. (2006). Social Network Typologies and Mental Health among Elder Adults. J Gerontol B Psychol Sci Soc Sci, 61(1), 25-32. doi: 10.1093/gerontonb/61.1

Fratiglioni, L., Paillard-Borg, S. y Winblad, B. (2004). An Active and Socially Integrated Lifestyle in Late Life Might Protect against Dementia. Lancet Neurol, 3, 343-353. doi: 10.1016/S1474-4422(04)00767-7

Garssen, B. (2004). Psychological Factors and Cancer Development: Evidence after 30 Years of Research. Clinical Psychology Review, 24, 315-338. doi: 10.1016/j. cpr.2004.01.002
Giles, L., Glonek, G., Luszcz, A. M. y Andrews, G. (2005). Efecto de las redes sociales de supervivencia de 10 años en los australianos muy antiguo: el estudio australiano longitudinal del envejecimiento. J Epidemiol Community Health, 59, 574-579. doi: 10.1136/jech.2004.025429

Heckman, T. G. (2003). The Chronic Illness Quality of Life (ciqol) Model: Explaining Life Satisfaction in People Living with hiv Disease. Health Psychology, 22, 140-147. doi: 10.1037/0278-6133.22.2.140

Huenchuan, S., Roqué, M. y Arias, C. (2009). Envejecimiento y sistemas de cuidado: ¿oportunidad o crisis? Santiago de Chile: Naciones Unidas.

Iacub, R. y Arias, C. (2011). Empoderamiento en la vejez. Journal of Behavior, Health and Social Issues, 2(2), 25-32.

Kahn, J. H., Hessling, R. M. y Russell, D. W. (2003). Social Support, Health, and Well-Being among the Elderly: What is the Role of Negative Affectivity? Personality and Individual Differences, 35, 5-17. doi: 10.1016/ S0191-8869(02)00135-6

Marco-Navarro, F. (2006). Cuidado no remunerado y acceso a la protección social. Nómadas, 24, 36-43.

Meléndez-Moran, J. C., Tomás-Miguel, J. M. y Navarro-Pardo, E. (2007). Análisis de las redes sociales en la vejez a través de la entrevista Manheim. Salud Pública de México, 49(6), 408-414.

Naciones Unidas (1998). Año Internacional de las Personas de Edad, 1999. Nueva York: Naciones Unidas.

Naciones Unidas. (2002). Informe de la Segunda Asamblea Mundial sobre Envejecimiento. Nueva York: Naciones Unidas.

O'Luanaigh, C. y Lawlor, B. A. (2008). Loneliness and the Health of Older People. Int J Geriatr Psychiatry, 23, 1213-1221.

Organización Mundial de la Salud (oms). (2002). Envejecimiento activo: Un marco político. Revista Española de Geriatría y Gerontología, 37(2), 74-105.

Phillips, D. R., Sui, O.L., Yeh, A. G. y Cheng, K. H. (2008). Informal Social Support and Older Person's Psychological Well Being in Hong Kong. Journal of Cross-Cultural Gerontology, 23(1), 39-55. 10.1007/s10823-007-9056-0

Prince-Paul, M. (2008). Understanding the Meaning of Social Well-Being at the End of Life. Oncology Nursing Forum, 35(3), 365-371. doi: 10.1188/08.ONF.365-371

Reblin, M. y Uchino, B. N. (2008). Social and Emotional Support and its Implication for Health. Curr Opin Psychiatry, 21, 201-205. doi: 10.1097/YCO.0b013e3282f3ad89

Reyes, C., Camacho, M., Eschbach, K. y Markides, K. (2006). El contexto de la familia y el vecindario en la salud de los ancianos del estudio epese hispano. Colombia Médica, 37, 45-49. 
Robles, L. (2003). Una vida cuidando a los demás: una "carrera" de vida en ancianas cuidadoras. Ponencia en el 51 Congreso Nacional de Americanistas. Santiago de Chile, Chile. Recuperado de http://www.redadultosmayores.com.ar/buscador/files/CALID019.PDF

Roqué, M. y Fassio, A. (2012). Gerontología comunitaria e institucional (3a ed.). Mar del Plata: Universidad Nacional de Mar del Plata (UNMDP) - Ministerio de Desarrollo Social.

Salinas, A., Manrique, B. y Téllez-Rojo, M. (2008). Redes de apoyo social en la vejez: adultos mayores beneficiarios del componente para Adultos Mayores del Programa Oportunidades. Ponencia en el III Congreso de la Asociación Latinoamericana de Población, alap. Córdoba, Argentina.

Shirom, A., Toker, S., Alkaly, Y., Jacobson, O. y Balicer, R. (2011). Work-Based Predictors of Mortality: A 20-Year Follow-Up of Healthy Employees. American Psychological Association, 30(3), 268-275. doi: 10.1037/a0023138

Sluzki, C. (1996). La red social: frontera de la práctica sistémica. Barcelona: Gedisa.

Uchino, B. N. (2006). Social Support and Health: A Review of Physiological Processes Potentially Underlying Links to Disease Outcomes. J Behav Med, 29, 377-387. doi: org/10.1007/s10865-006-9056-5
Vanderhorst, R. K. y McLaren, S. (2005). Social Relationship as Predictors of Depression and Suicidal Ideation in Older Adults. Aging and Mental Health, 9(6), 517-525. 10.1080/13607860500193062

Vaux, A. y Harrison, D. (1985). Support Network Characteristics Associated with Support Satisfaction and Perceived Support. American Journal of Community Psychology, 13(3), 245-268. doi: 10.1007/BF00914932

Vivaldi, F. y Barra, E. (2012). Bienestar psicológico, apoyo social percibido y percepción de salud en adultos mayores. Terapia psicológica, 30(2), 23-29.

Waern, M., Rubenowitz, E. y Wilhelmson, K. (2003). Predictors of Suicide in the Old Elderly. Gerontology, 49, 328-334. doi: 10.1159/000071715

Yoon, D. y Lee, E. (2007). The Impact of Religiousness, Spiritually and Social Support on Psychological Well-Being among Older Adults in Rural Areas. Journal of Gerontological Social Work, 48(3/4), 281-298. doi: 10.1300/ J083N48N03_01

Zinbarg, R., Lee, J. E. y Yoon, L. (2007). Dyadic predictors of outcome in a cognitive-behavioral program for patients with Generalized Anxiety Disorder in committed relationships: A 'spoonful of sugar' and a dose of non-hostile criticism may help. Behaviour Research and Therapy, 45, 699-713. doi: 10.1016/j.brat.2006.06.005 